\title{
Effect of plasma vitamin C levels on Parkinson's disease and age at onset: a Mendelian randomization study
}

\author{
Haijie Liu' ${ }^{1}$, Yan Zhang ${ }^{2}$, Haihua Zhang ${ }^{3}$, Longcai Wang ${ }^{4}$, Tao Wang ${ }^{5,6}$, Zhifa Han ${ }^{7}$, Liyong Wu ${ }^{1 *}$ and \\ Guiyou Liu ${ }^{3,6,8,9^{*}}$ (D)
}

\begin{abstract}
Background: Until now, epidemiological evidence regarding the association between vitamin $\mathrm{C}$ intake (both diet and supplements) and Parkinson's disease (PD) remains inconsistent. Hence, it is necessary to establish the causal link between vitamin C levels and PD, and further develop effective therapies or prevention.

Methods: We selected 11 newly identified plasma vitamin $C$ genetic variants from a large-scale plasma vitamin $C$ GWAS dataset $(n=52,018)$ as the effective instrumental variables, and extracted their corresponding GWAS summary statistics from PD (33,674 PD cases and 449,056 controls) and PD age at onset (AAO) $(n=28,568)$. We then performed a Mendelian randomization (MR) study to evaluate the causal association of plasma vitamin C levels with PD and PD AAO using inverse-variance weighted (IVW), the weighted median, MR-Egger, and MR-PRESSO test.
\end{abstract}

Results: We did not observe any significant association between genetically increased vitamin C levels and PD. Interestingly, we found a reduced trend of PD AAO (1.134 years) with 1 SD genetically increased vitamin C levels using IVW (beta $=-1.134,95 \% \mathrm{Cl}:[-2.515,0.248], P=0.108)$. Importantly, this trend was further successfully verified using both weighted median and MR-Egger. Each 1 SD genetically increased vitamin C levels could reduce PD AAO 1.75 and 2.592 years using weighted median (beta $=-1.750,95 \% \mathrm{Cl}:[-3.396,-0.105], P=0.037$ ) and MR-Egger (beta $=-$ $2.592,95 \% \mathrm{Cl}:[-4.623,-0.560], P=0.012)$.

Conclusions: We demonstrated the causal association between genetically increased plasma vitamin $C$ levels and reduced PD AAO in people of European descent. Randomized controlled trials are required to clarify whether diet intake or supplement, or both could reduce the AAO of PD.

Keywords: Parkinson's disease, Vitamin C, Genome-wide association study, Mendelian randomization, Inversevariance weighted

*Correspondence: wmywly@hotmail.com; liuguiyou1981@163.com 1 Department of Neurology, Xuanwu Hospital, Capital Medical University, Beijing 100053, China

${ }^{3}$ Beijing Institute of Brain Disorders, Laboratory of Brain Disorders, Ministry of Science and Technology, Collaborative Innovation Center for Brain Disorders, Capital Medical University, Beijing 100069, China Full list of author information is available at the end of the article

\section{Background}

Parkinson's disease (PD) is the second most common neurodegenerative disease in the elderly [1,2]. Evidence shows that oxidative stress is involved in the degeneration of dopaminergic neurons in PD [3]. Vitamin $\mathrm{C}$ is a major antioxidant and a neuromodulator in dopaminergic neurons, which could neutralize reactive oxygen species and reduce oxidative stress $[4,5]$. Observational study indicated significantly reduced lymphocyte vitamin $\mathrm{C}$ levels

c) The Author(s) 2021. This article is licensed under a Creative Commons Attribution 4.0 International License, which permits use, sharing, adaptation, distribution and reproduction in any medium or format, as long as you give appropriate credit to the original author(s) and the source, provide a link to the Creative Commons licence, and indicate if changes were made. The images or other third party material in this article are included in the article's Creative Commons licence, unless indicated otherwise in a credit line to the material. If material is not included in the article's Creative Commons licence and your intended use is not permitted by statutory regulation or exceeds the permitted use, you will need to obtain permission directly from the copyright holder. To view a copy of this licence, visit http://creativeco mmons.org/licenses/by/4.0/. The Creative Commons Public Domain Dedication waiver (http://creativecommons.org/publicdomain/ zero/1.0/) applies to the data made available in this article, unless otherwise stated in a credit line to the data. 
in patients with severe PD compared with less severe PD patients [5]. Meanwhile, a reduced trend in plasma vitamin C levels in patients with severe PD was also reported [5]. These findings show that high vitamin $C$ intake (both diet and supplements) may be theoretically beneficial for PD treatment or prevention.

Until now, epidemiological evidence regarding the association between vitamin $\mathrm{C}$ intake (both diet and supplements) and PD remains inconsistent. In 1997, the community-based Rotterdam Study in the Netherlands indicated that high dietary intake of vitamin $C$ could not decrease the risk of PD with odds ratio $(\mathrm{OR})=0.9(95 \%$ confidence interval (CI): 0.4-1.9) per 100-mg vitamin $C$ intake [6]. In 2002, the Nurses' Health Study and the Health Professionals Follow-Up Study identified that none of the total vitamin intake, vitamin $\mathrm{C}$ supplement, and dietary vitamin $\mathrm{C}$ intake, was significantly associated with the risk of PD [7]. In 2011, a Japan multicenter hospital-based case control study indicated that higher dietary intake of vitamin $C$ was not associated with the decreased risk of PD [8]. In 2016, the Nurses' Health Study and the Health Professionals Follow-up Study showed that vitamin $C$ intake from diet could significantly reduce the risk of PD [9]. However, this significant association was not successfully replicated in a 4-year lag analysis [9]. Meanwhile, the combined vitamin $C$ intake from diet and supplements was not associated with the PD risk [9]. In 2017, the Swedish Mammography Cohort
(SMC) and the Cohort of Swedish Men (COSM) study found that dietary vitamin $C$ intake was inversely associated with PD risk in women (HR $=0.91,95 \%$ CI: 0.831.00) [10]. In 2021, the Swedish National March Cohort study (43,865 men and women aged 18-94 years with a mean follow-up time of 17.6 years) found that individuals with the highest dietary vitamin $\mathrm{C}$ had the reduced $\mathrm{PD}$ risk (hazard ratio $(\mathrm{HR})=0.68$; 95\% CI: $0.52-0.89$ ) compared with those the lowest dietary vitamin C [11].

Hence, the causal link between vitamin $C$ levels and PD remains unclear. In recent years, Mendelian randomization (MR) design has been widely used to determine the causal inferences and could overcome the methodological limitations of observational studies [12]. Here, we performed a MR study to investigate the causal association between plasma vitamin $C$ levels and PD using multiple large-scale genome-wide association study (GWAS) datasets from plasma vitamin $\mathrm{C}, \mathrm{PD}$ and $\mathrm{PD}$ age at onset (AAO) [13-15].

\section{Methods \\ Study design}

This MR study is based on the large-scale GWAS summary datasets in plasma vitamin $\mathrm{C}, \mathrm{PD}$ and $\mathrm{PD}$ AAO [13-15]. All participants have given informed consents in all these corresponding original studies $[14,15]$. In general, MR must meet three principal assumptions, as provided in Fig. 1, a flow chart about our MR study design.

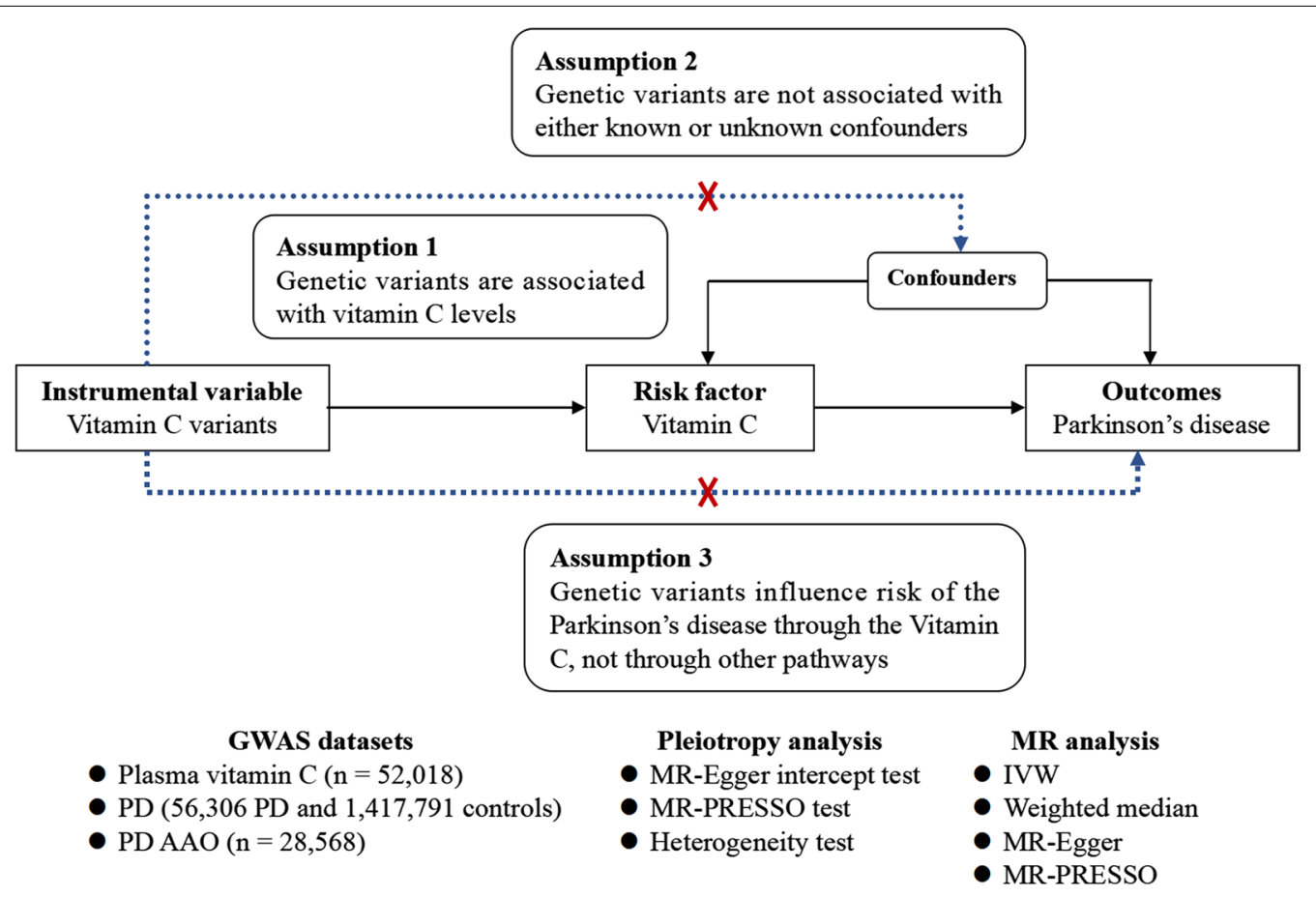

Fig. 1 The flow chart about the MR study design 
The second and third assumptions are collectively known as independence from pleiotropy, as described in recent studies [16-18].

\section{Plasma vitamin C genetic variants}

Typically, independent genetic variants with genomewide significance $\left(P<5 \times 10^{-8}\right)$ are selected as the potential instruments in MR studies, as described in recent studies [19-22]. Here, we selected 11 independent genetic variants with a genome-wide significant level $\left(P<5 \times 10^{-8}\right)$ from a recent plasma vitamin C GWAS dataset in 52,018 individuals of European ancestry [13]. This GWAS is based on a large-scale meta-analysis in four populations including the Fenland study, European Prospective Investigation into Cancer and Nutrition (EPIC)-InterAct study, EPIC-Norfolk study, and EPICCVD study [13]. The summary results regarding the effect of each genetic variant on vitamin $C$ levels and the standard errors were provided in Table 1.

\section{PD GWAS dataset}

The PD GWAS dataset is from the International Parkinson's Disease Genomics Consortium (IPDGC) that conducted the large-scale meta-analysis of 17 GWAS datasets in 56,306 PD cases (37,688 PD cases, 18,618 UK Biobank proxy-cases) and 1,417,791 control individuals of European ancestry [14]. However, the GWAS summary statistics from the meta-analysis of all these selected 17 GWAS datasets are not publicly available. Hence, we selected the subgroup of these 17 GWAS datasets including 14 GWAS datasets by excluding Nalls and colleagues, 23 and Me post-Chang and colleagues and Web-Based Study of Parkinson's disease [14]. The subgroup GWAS dataset included 33,674 PD cases and 449,056 controls [14]. Table 2 provides the demographic profiles about the 14 PD GWAS datasets, as provided in the original study [14].

\section{PD AAO GWAS dataset}

The PD AAO GWAS dataset is from the large-scale meta-analysis of 18 PD AAO GWAS datasets in 28,568 PD cases including 17 independent cohorts from IPDGC $(\mathrm{n}=17,996)$ and the 23andMe PD cohort $(\mathrm{n}=10,572)$ [15]. The average AAO in the IPDGC dataset was 62.14 (range $20-96, \mathrm{SD}=12.08$ ), and average $\mathrm{AAO}$ in the 23andMe dataset was 60.71 (range $40-97, \mathrm{SD}=9.98$ ) [15]. Table 3 provides the demographic profiles about the 18 PD AAO GWAS datasets, as provided in the original study [15].

\section{Pleiotropy analysis}

The pleiotropy analysis is based on three different statistical methods including MR-Egger intercept test [28], MR pleiotropy residual sum and outlier (MR-PRESSO) global test [28], and heterogeneity test using Cochran's Q statistic and $I^{2}$ statistic $[29,30]$. The significance threshold is $P<0.05$. All the statistical tests were completed using three $\mathrm{R}$ Packages including 'meta: General Package for Meta-Analysis', 'MendelianRandomization' and 'MRPRESSO', respectively [12].

\section{MR analysis}

We selected four MR analysis methods including the inverse-variance weighted (IVW), the weighted median, MR-Egger, and MR-PRESSO test [12, 28, 31]. The effect size (beta) and 95\% confidence interval (CI) correspond to 1 standard deviation (SD) in vitamin $C$ levels. All the statistical tests were completed using R Packages 'MendelianRandomization' and 'MR-PRESSO', respectively [12]. The significance threshold is $P<0.05$.

Table 1 Main characteristics of 11 selected plasma vitamin C genetic variants

\begin{tabular}{|c|c|c|c|c|c|c|c|c|c|c|}
\hline SNP & Chromosome & Position (GRCh37) & EA & NEA & EAF & Beta & SE & $P$ value & Gene & $R^{2(\%)}$ \\
\hline rs6693447 & 1 & 2330190 & $\mathrm{~T}$ & G & 0.551 & 0.039 & 0.006 & $6.25 E-10$ & RER1 & 0.08 \\
\hline rs13028225 & 2 & 220031255 & $\mathrm{~T}$ & C & 0.857 & 0.102 & 0.009 & $2.38 \mathrm{E}-30$ & $S L C 23 A 3$ & 0.2 \\
\hline rs33972313 & 5 & 138715502 & $C$ & $\mathrm{~T}$ & 0.968 & 0.36 & 0.018 & $4.61 \mathrm{E}-90$ & $S L C 23 A 1$ & 0.76 \\
\hline rs 10051765 & 5 & 176799992 & $C$ & $\mathrm{~T}$ & 0.342 & 0.039 & 0.007 & $3.64 \mathrm{E}-09$ & RGS14 & 0.06 \\
\hline rs7740812 & 6 & 52725787 & G & A & 0.594 & 0.038 & 0.006 & $1.88 \mathrm{E}-09$ & GSTA5 & 0.08 \\
\hline rs 174547 & 11 & 61570783 & $C$ & $\mathrm{~T}$ & 0.328 & 0.036 & 0.007 & $3.84 \mathrm{E}-08$ & FADS1 & 0.05 \\
\hline rs117885456 & 12 & 96249111 & A & G & 0.087 & 0.078 & 0.012 & $1.70 E-11$ & SNRPF & 0.08 \\
\hline rs 2559850 & 12 & 102093459 & A & G & 0.598 & 0.058 & 0.006 & $6.30 E-20$ & CHPT1 & 0.18 \\
\hline rs10136000 & 14 & 105253581 & A & G & 0.283 & 0.04 & 0.007 & $1.33 \mathrm{E}-08$ & AKT1 & 0.06 \\
\hline rs56738967 & 16 & 79740541 & $C$ & G & 0.321 & 0.041 & 0.007 & $7.62 \mathrm{E}-10$ & MAF & 0.07 \\
\hline rs9895661 & 17 & 59456589 & $\mathrm{~T}$ & C & 0.817 & 0.063 & 0.008 & $1.05 E-14$ & BCAS3 & 0.12 \\
\hline
\end{tabular}

Beta is the regression coefficient based on the vitamin $C$ raising allele (effect allele); $R^{2}$, the proportion of vitamin $C$ variance explained by the selected genetic variants SNP single-nucleotide polymorphism, EA effect allele, NEA non-effect allele, EAF effect allele frequency, SE standard error 
Table 2 Demographic profiles about the PD GWAS dataset

\begin{tabular}{|c|c|c|c|c|c|c|}
\hline Study & Cases & Controls & Female cases (\%) & $\begin{array}{l}\text { Female } \\
\text { control } \\
(\%)\end{array}$ & $\begin{array}{l}\text { Case age at } \\
\text { onset (mean, } \\
\text { SD) }\end{array}$ & $\begin{array}{l}\text { Control age at } \\
\text { last exam (mean, } \\
\text { SD) }\end{array}$ \\
\hline Baylor College of Medicine/University of Maryland & 769 & 195 & 33.81 & 69.74 & $64.83(10.12)$ & $65.48(8.31)$ \\
\hline Finnish Parkinson's & 386 & 493 & 45.85 & 78.9 & $55.27(5.64)$ & $92.35(3.86)$ \\
\hline Harvard Biomarker Study (HBS) & 527 & 472 & 34.35 & 61.65 & $66.31(10.07)$ & $69.9(9.02)$ \\
\hline McGill Parkinson's & 582 & 905 & 34.54 & 48.4 & $65.71(9.79)$ & $55.79(10.69)$ \\
\hline Oslo Parkinson's Disease Study & 476 & 462 & 35.71 & 42.21 & $65.32(9.28)$ & $61.85(11.06)$ \\
\hline Parkinson's Disease Biomarker's Program (PDBP) & 512 & 282 & 38.67 & 51.06 & $64.46(9.37)$ & $62.19(10.73)$ \\
\hline Parkinson's Progression Markers Initiative (PPMI) & 363 & 165 & 33.06 & 33.33 & $64.24(9.65)$ & $63.79(10.59)$ \\
\hline System Genomics of Parkinson's Disease (SGPD) & 1169 & 968 & 35.24 & 53.93 & $59.88(10.86)$ & $66.64(9.65)$ \\
\hline Spanish Parkinson's (IPDGC) & 2110 & 1333 & 43.13 & 54.39 & $63.92(12.54)$ & $64.03(12.59)$ \\
\hline Tubingen Parkinson's disease cohort (CouragePD) & 666 & 542 & 36.04 & 57.93 & $59.89(11.25)$ & $67.48(8.41)$ \\
\hline Vance (dbGap phs000394) & 620 & 299 & 27.74 & 50.84 & $77.47(8.40)$ & $81.98(12.78)$ \\
\hline UKPDMED (CouragePD) & 1025 & 655 & 32.78 & 72.67 & NA & NA \\
\hline UKBioBank & 18,618 & 436,419 & 57.62 & 54.14 & $58.45(7.20)$ & $56.69(8.05)$ \\
\hline NeuroX_dbGaP (phs000918.v1.p1) & 5851 & 5866 & NA & NA & NA & NA \\
\hline All & 33,674 & 449,056 & NA & NA & NA & NA \\
\hline
\end{tabular}

Table 3 Demographic profiles about the PD AAO GWAS dataset

\begin{tabular}{lccc}
\hline Dataset & PD cases & $\begin{array}{l}\text { Average age of onset of cases } \\
\text { (range) }\end{array}$ & $\begin{array}{l}\text { Sex ratio male/ } \\
\text { female of cases }\end{array}$ \\
\hline Dutch GWAS [23] & 764 & $54.94(21-84)$ & 1.74 \\
Finnish GWAS & 377 & $55.27(30-66)$ & 1.19 \\
German GWAS [24] & 663 & $55.84(28-86)$ & 1.55 \\
Harvard Biomarker Study (HBS) & 525 & $66.31(35-89)$ & 1.92 \\
McGill Parkinson's & 580 & $65.56(37-91)$ & 1.89 \\
IPDGC NeuroX [25] & 5428 & 1.82 \\
NIA PD GWAS [24] & 845 & $61.27(20-89)$ & 1.46 \\
OsloParkinson's Disease Study & 476 & $58.25(20-87)$ & 1.8 \\
Parkinson's Disease Biomarker's Program (PDBP) & 512 & $55.70(24-83)$ & 1.59 \\
Parkinson's Progression Markers Initiative (PPMI) & 360 & $64.46(34-87)$ & 2.03 \\
PROBAND & 1815 & $64.24(36-87)$ & 1.85 \\
PROPARK & 235 & $66.25(29-90)$ & 2.09 \\
Baylor College of Medicine/University of Maryland & 764 & $55.69(29-81)$ & 1.95 \\
Spanish GWAS [26] & 1928 & $64.83(23-92)$ & 1.35 \\
Tuebingen Parkinson's Disease cohort & 666 & $63.90(20-95)$ & 1.78 \\
WTCCC PD GWAS [27] & 1477 & $59.89(23-87)$ & 1.6 \\
System Genomics of Parkinson's disease (SGPD) & 581 & $64.10(23-96)$ & 1.75 \\
Total IPDGC & 17,996 & $59.96(24-84)$ & 1.7 \\
23 and Me & 10,572 & $62.14(20-96)$ & 1.54 \\
Total & 28,568 & $60.71(40-97)$ & 1.64 \\
\hline
\end{tabular}




\section{Power analysis}

The proportion of vitamin $C$ variance explained by the selected genetic variants $R^{2}$.

$$
R^{2}=\sum_{i=1}^{K} \frac{\beta_{i}^{2}}{\beta_{i}^{2}+2 * N * \operatorname{se}\left(\beta_{i}\right)^{2}}
$$

Here, $\beta_{i}$ is the effect size for $S N P_{i}$, se $\left(\beta_{i}\right)$ is the standard error for $S N P_{i}, N$ is the sample size for $S N P_{i}$, and $K$ is the number of the selected genetic variants [32]. The statistical power is calculated using the web-based tool mRnd and a two-sided type-I error rate $\alpha$ of 0.05 [33].

\section{Results}

\section{Vitamin C genetic variants with PD and PD AAO}

We successfully extracted the summary statistics corresponding to the 11 vitamin $C$ genetic variants in $\mathrm{PD}$ and PD AAO GWAS datasets, respectively. It is noted that rs56738967 (C/G, C with the minor allele frequency $(\mathrm{MAF})=0.321)$ is an ambiguous palindromic variant (i.e. with alleles either $A / T$ or $\mathrm{C} / \mathrm{G}$ ). Hence, we selected the allele frequency to distinguish the effect allele in both GWAS datasets. More detailed information about the association of these 11 vitamin $\mathrm{C}$ genetic variants with PD and PD AAO is proved in Table 4.

\section{Pleiotropy analysis}

We did not identify any significant pleiotropic variant among the selected 11 vitamin $C$ genetic variants in both the PD and PD AAO GWAS datasets using the three statistical methods with all $P$ values $>0.05$. More detailed pleiotropy analysis results are provided in Table 5 . Hence, all these selected 11 vitamin $C$ genetic variants could be taken as the effective instrumental variables in MR analysis.

\section{MR analysis}

In PD GWAS dataset, we did not observe any significant association between genetically increased vitamin $\mathrm{C} \mathrm{lev-}$ els and PD risk using all the four selected MR methods, as described in Table 6 . Interestingly, we found a reduced trend of PD AAO (1.134 years) with 1 SD genetically increased vitamin $C$ levels using IVW (beta $=-1.134$, 95\% CI: $[-2.515,0.248], P=0.108)$. Importantly, this reduced trend was further successfully verified using both weighted median and MR-Egger. In brief, each 1

Table 4 Association of 11 vitamin C genetic variants in PD and PD AAO

\begin{tabular}{|c|c|c|c|c|c|c|c|c|c|}
\hline \multirow[t]{2}{*}{ SNP } & \multicolumn{3}{|c|}{ Plasma vitamin C GWAS } & \multicolumn{3}{|c|}{ PD GWAS } & \multicolumn{3}{|c|}{ PD AAO GWAS } \\
\hline & EA & NEA & EAF & Beta & SE & $P$ value & Beta & SE & $P$ value \\
\hline rs10051765 & C & $\mathrm{T}$ & 0.342 & 0.013 & 0.019 & 0.477 & 0.104 & 0.125 & 0.405 \\
\hline rs10136000 & A & G & 0.283 & 0.003 & 0.021 & 0.872 & 0.100 & 0.141 & 0.478 \\
\hline rs117885456 & A & G & 0.087 & -0.053 & 0.043 & 0.216 & 0.274 & 0.267 & 0.305 \\
\hline rs13028225 & $\mathrm{T}$ & C & 0.857 & 0.055 & 0.025 & 0.024 & -0.325 & 0.167 & 0.051 \\
\hline rs174547 & C & $\mathrm{T}$ & 0.328 & 0.003 & 0.018 & 0.853 & 0.141 & 0.121 & 0.243 \\
\hline rs2559850 & $A$ & G & 0.598 & -0.026 & 0.023 & 0.247 & 0.013 & 0.141 & 0.929 \\
\hline rs33972313 & C & $\mathrm{T}$ & 0.968 & -0.006 & 0.052 & 0.903 & -0.751 & 0.353 & 0.033 \\
\hline rs56738967 & C & $\mathrm{G}$ & 0.321 & -0.034 & 0.019 & 0.063 & -0.003 & 0.125 & 0.980 \\
\hline rs6693447 & $\mathrm{T}$ & G & 0.551 & -0.034 & 0.019 & 0.066 & -0.049 & 0.125 & 0.697 \\
\hline rs7740812 & G & A & 0.594 & -0.034 & 0.023 & 0.135 & -0.238 & 0.143 & 0.096 \\
\hline rs9895661 & $\mathrm{T}$ & C & 0.817 & -0.002 & 0.023 & 0.931 & 0.032 & 0.151 & 0.833 \\
\hline
\end{tabular}

Beta is the regression coefficient based on the vitamin $C$ raising allele (effect allele)

$S N P$ single-nucleotide polymorphism, $E A$ effect allele, $N E A$ non-effect allele, $E A F$ effect allele frequency, $S E$ standard error

Table 5 Pleiotropy analysis of 11 selected plasma vitamin C genetic variants

\begin{tabular}{|c|c|c|c|c|c|c|c|}
\hline \multirow[t]{2}{*}{ GWAS dataset } & \multicolumn{3}{|c|}{ MR-Egger intercept } & \multirow{2}{*}{$\begin{array}{l}\text { MR-PRESSO } \\
P \text { value }\end{array}$} & \multicolumn{3}{|c|}{ Heterogeneity test } \\
\hline & intercept & $95 \% \mathrm{Cl}$ & $P$ value & & $I^{2}(\%)$ & $95 \% \mathrm{Cl}$ & $Q P$ value \\
\hline PD & -0.016 & {$[-0.043,0.011]$} & 0.243 & 0.08 & 42.4 & {$[0.0 \% ; 71.5 \%]$} & 0.0669 \\
\hline PD AAO & 0.127 & {$[-0.011,0.266]$} & 0.072 & 0.271 & 15.6 & {$[0.0 \% ; 56.1 \%]$} & 0.2951 \\
\hline
\end{tabular}

The significance threshold is $P<0.05$ 
Table 6 The causal association of plasma vitamin C levels with $P D$ and PD AAO

\begin{tabular}{lllll}
\hline GWAS dataset & Method & Beta & $\mathbf{9 5 \%} \mathbf{C l}$ & $\boldsymbol{P}$ value \\
\cline { 2 - 5 } & IVW & -0.048 & {$[-0.296,0.201]$} & 0.708 \\
Weighted & -0.018 & {$[-0.272,0.237]$} & 0.893 \\
median & & & \\
MR-Egger & 0.130 & {$[-0.255,0.516]$} & 0.508 \\
PD AAO & MR-PRESSO & -0.048 & {$[-0.296,0.201]$} & 0.716 \\
& IVW & -1.134 & {$[-2.515,0.248]$} & 0.108 \\
& Weighted & -1.750 & {$[-3.396,-$} & $\mathbf{0 . 0 3 7}$ \\
median & & $0.105]$ & \\
& MR-Egger & -2.592 & {$[-4.623,-$} & $\mathbf{0 . 0 1 2}$ \\
& MR-PRESSO & -1.134 & {$[-2.515,0.248]$} & 0.139
\end{tabular}

The significance of association between vitamin $C$ levels and AD was at $P<0.05$; The significant $P$ values 0.037 and 0.012 were bold

$C /$ confidence interval, IVW inverse-variance weighted, MR-PRESSO Mendelian randomization pleiotropy residual sum and outlier

SD genetically increased vitamin $C$ levels could reduce PD AAO 1.75 and 2.592 years using weighted median (beta $=-1.750,95 \% \mathrm{CI}$ : $[-3.396,-0.105], P=0.037$ ) and MR-Egger (beta $=-2.592,95 \%$ CI: $[-4.623,-$ $0.560], P=0.012$ ). These estimates were consistent in terms of direction and magnitude, as provided in Table 6 . Figure 2 shows the individual MR estimates about the causal effect of vitamin $\mathrm{C}$ levels on PD AAO using MREgger method.

\section{Power analysis}

All these selected 11 genetic variants could explain 1.79\% variance of plasma vitamin C levels. Our MR study had $80 \%$ power to detect an OR of 0.91 or lower per SD increase in vitamin $\mathrm{C}$ levels for PD. In order to calculate the power about the causal association between vitamin $\mathrm{C}$ levels and PD AAO, the regression coefficients from a observational study to evaluate the association between plasma vitamin C levels and PD AAO are needed including both the without confounder-adjustment and with confounder-adjustment [33]. However, this kind of observational study is not publicly available until now. Hence, we could not evaluate the power about the MR analysis in PD AAO.

\section{Discussion}

Until now, epidemiological evidence about the association between vitamin $C$ intake and PD remains inconsistent [6-11]. Hence, it is necessary to establish the causal link between vitamin $C$ levels and PD, and to develop effective therapies or prevention. Hence, we selected 11 vitamin $C$ genetic variants as the effective instrumental variables and extracted their corresponding summary statistics in large-scale PD GWAS and PD AAO datasets, respectively. We then performed a MR study to evaluate the causal association of vitamin $C$ levels with $\mathrm{PD}$ and $\mathrm{PD}$ AAO. We found no causal association between genetically increased vitamin $C$ levels and PD risk. Interestingly,

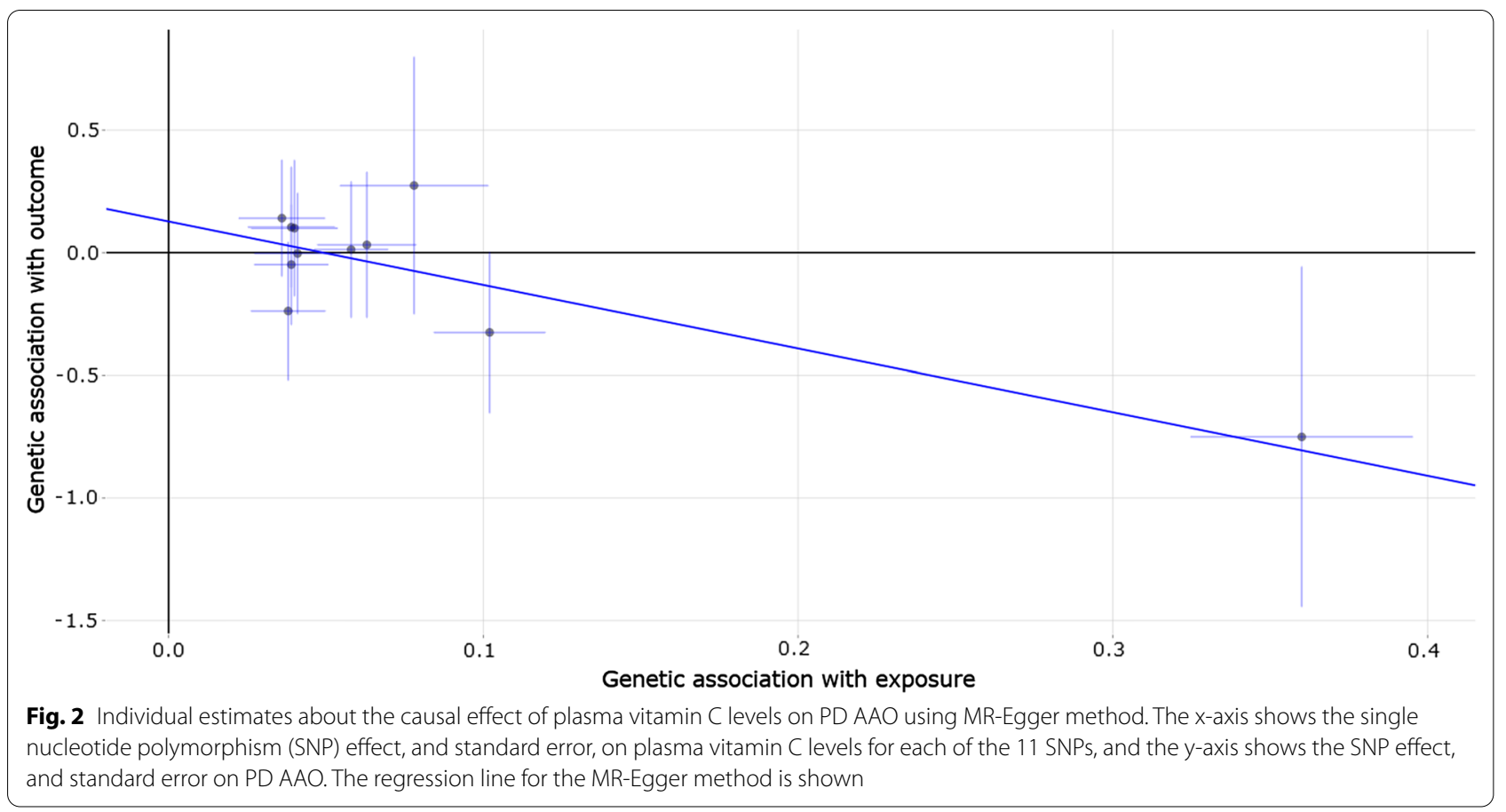


we found that genetically increased vitamin $C$ levels were significantly associated with reduced PD AAO. Each 1 SD genetically increased vitamin $C$ levels could reduce PD AAO 1.134, 1.75 and 2.592 years using IVW, weighted median and MR-Egger methods, respectively.

It is noted that our findings only reflect the effect of plasma vitamin $\mathrm{C}$ levels on $\mathrm{PD}$ and its age at onset, but not the serum vitamin $C$ levels. Until now, two observational studies have been performed to evaluate the association of serum vitamin $\mathrm{C}$ levels with the AAO, duration and progression of PD [34, 35]. Fernandez-Calle and colleagues compared the serum vitamin $C$ levels using 63 PD patients using their spouses as the control group [35]. The serum levels of vitamin $C$ did not show significant difference in between PD and controls [35]. Meanwhile, they found no correlation of serum vitamin $\mathrm{C}$ levels with the AAO, duration and progression (scores of the Unified PD Rating Scale or the Hoehn and Yahr staging) of PD [35]. King and colleagues measured the serum vitamin $\mathrm{C}$ levels in $27 \mathrm{PD}$ patients and 16 age-matched control subjects [34]. They found significantly increased serum vitamin C levels in PD cases than the controls [34]. Meanwhile, there was no correlation of serum vitamin C levels with the duration or progression of PD [34].

Until now, there is not publicly available observational evidence that high plasma vitamin $C$ levels could reduce the PD AAO. However, there is at least one study that had evaluated the association of plasma and lymphocyte $\mathrm{C}$ levels with the progression of PD (determined by the Hoehn-Yahr scale) using 62 PD cases [5]. The results indicated that plasma vitamin $\mathrm{C}$ levels tended to be lower in severe PD patients compared with those at less severe stages (OR, 0.98; 95\% CI 0.96-1.00; $P=0.09$ ) [34]. Meanwhile, the lymphocyte vitamin $C$ levels were significantly lower in severe PD patients (OR $=0.87,95 \%$ CI 0.80 $0.97 ; P<0.01)$ compared with those at less severe stages [5].

It is known that vitamin $C$ contributes to many health benefits especially antioxidant properties $[36,37]$. The US recommended dosage of vitamin $C$ is $100-120 \mathrm{mg} /$ day for adults [36]. However, vitamin $\mathrm{C}$ is also a pro-oxidative factor [37, 38]. Vitamin $C$ could be readily oxidized, which further causes DNA damage and produce oxidative stress $[37,38]$. In order to translate these genetic findings into clinical and public health implications, randomized controlled trials are required to assess the effect of plasma vitamin $\mathrm{C}$ levels on PD AAO, and further clarify whether diet intake or supplement, or both could reduce the AAO of $\mathrm{PD}$.

Our MR study may have several strengths. First, this MR design was based on the large-scale plasma vitamin C GWAS dataset $(n=52,018)$ and large-scale PD GWAS dataset (33,674 PD cases and 449,056 controls) and PD
AAO GWAS dataset $(n=28,568)$. Importantly, the individuals from all these three GWAS datasets are of European ancestry, which contribute to reduce the influence of population stratification. Second, we selected 11 independent genetic variants as the potential instruments, and further demonstrated all these selected 11 genetic variants to be the effective instrumental variables using three independent statistical methods. Third, we selected four MR methods including IVW, weighted median, MREgger, and MR-PRESSO. Importantly, all these four MR methods produce consistent estimates.

\section{Limitations}

Our MR study may also have some limitations. First, our MR analysis just reflects the findings in European ancestry. The causal association between vitamin $\mathrm{C} \mathrm{lev-}$ els and AD risk may be different across different ancestries. Hence, our findings should be further replicated in other ancestries. Second, the GWAS dataset from IPDGC is based on the clinically diagnosed PD and selfreport PD-by-proxy, respectively. Hence, there may be some differences across the different diagnostic criteria. Hence, PD GWAS dataset based the clinically diagnosed criteria should further verify our findings. Third, we demonstrated that the increased plasma vitamin $C$ levels could reduce the PD AAO. However, it remains unclear about the potential mechanisms underlying this causal association, which deserves to be thoroughly evaluated. Fourth, it is known that the sodium-dependent vitamin C transporters of the SLC23 family genes including SLC23A1 and SLC23A2 are involved in direct transport and regulation of vitamin $C$ concentrations [13]. Fortunately, genetic variants associated with plasma vitamin $C$ levels of genome-wide significance $\left(\mathrm{P}<5 \times 10^{-8}\right)$ at SLC23A1 (rs33972313, the strongest signal) and SLC23A3 (rs13028225, the second strongest signal) were successfully reported in the original GWAS dataset, as provided in Table 1 [13]. However, the original GWAS did not identify any genetic variant with genome-wide significance at $S L C 23 A 2$ [13]. Hence, we could not select the genetic variants at $S L C 23 A 2$ as the potential instrumental variables using the genome-wide significance criteria. One possible reason is that SVCT2 encoded by $S L C 23 A 2$ mainly regulates tissue levels of vitamin C, which further causes its impact on circulating vitamin $\mathrm{C}$ to be minimal $[13,39]$. The other is that the current sample size from the GWAS $(n=52,018)$ is not enough. Fifth, the genetically increased plasma vitamin $C$ level does not necessarily reflect in the plasma, as the equivocal results were reported in serum vitamin $\mathrm{C}$ and its association with PD [34, 35]. Hence, our findings deserve further investigation. 


\section{Conclusions}

In summary, our MR analysis demonstrated the causal association between genetically increased plasma vitamin $\mathrm{C}$ levels and reduced PD AAO in people of European descent. Hence, maintaining adequate plasma vitamin $\mathrm{C}$ levels may contribute to reduce the AAO of PD. Meanwhile, additional studies are also required to further verify our findings.

\section{Abbreviations \\ PD: Parkinson's disease; MR: Mendelian randomization; GWAS: Genome- wide association study; IGAP: International Genomics of Alzheimer's Project; MR-PRESSO: MR pleiotropy residual sum and outlier; IVW: Inverse-variance weighted.}

\section{Acknowledgements}

We thank the International Parkinson's Disease Genomics Consortium (IPDGC) for providing summary results data for these analyses.

\section{Authors' contributions}

GYL and YZ conceived and initiated the project. GYL, HHZ, ZFH, TW and YH analyzed the data, and wrote the first draft of the manuscript. All authors contributed to the interpretation of the results and critical revision of the manuscript for important intellectual content and approved the final version of the manuscript. All author read and approved the final manuscript.

\section{Funding}

This work was supported by funding from the National Natural Science Foundation of China (Grant No. 82071212 and 81901181), the Mathematical Tianyuan Fund of the National Natural Science Foundation of China (Grant No. 12026414), and Beijing Ten Thousand Talents Project (Grant No. 2020A15). This work was also partially supported by funding from the Science and Technology Beijing One Hundred Leading Talent Training Project (Z141107001514006), the Beijing Municipal Administration of Hospitals' Mission Plan (SML20150802), the Funds of Academic Promotion Programme of Shandong First Medical University \& Shandong Academy of Medical Sciences (No. 2019QL016, No. 2019PT007)

\section{Availability of data and materials}

All relevant data are within the paper. The authors confirm that all data underlying the findings are either fully available without restriction through consortia websites, or may be made available from consortia upon request.

\section{Declarations}

\section{Ethics approval and consent to participate}

This article contains human participants collected by several studies performed by previous studies. All participants gave informed consent in all the corresponding original studies, as described in the Methods. Here, our study is based on the large-scale GWAS datasets, and not the individual-level data. Hence, ethical approval was not applicable.

\section{Consent to participate}

Not applicable.

\section{Consent for publication}

Not applicable.

\section{Competing interests}

The authors declare no competing interests.

\section{Author details}

${ }^{1}$ Department of Neurology, Xuanwu Hospital, Capital Medical University, Beijing 100053, China. ${ }^{2}$ Department of Pathology, The Affiliated Hospital of Weifang Medical University, Weifang 261053, China. ${ }^{3}$ Beijing Institute of Brain Disorders, Laboratory of Brain Disorders, Ministry of Science and Technology,
Collaborative Innovation Center for Brain Disorders, Capital Medical University, Beijing 100069, China. ${ }^{4}$ Department of Anesthesiology, The Affiliated Hospital of Weifang Medical University, Weifang 261053, China. ${ }^{5}$ Academy for Advanced Interdisciplinary Studies, Peking University, Beijing, China. ${ }^{6}$ Chinese Institute for Brain Research, Beijing, China. ${ }^{7}$ School of Medicine, School of Pharmaceutical Sciences, THU-PKU Center for Life Sciences, Tsinghua University, Beijing, China. ${ }^{8}$ Beijing Key Laboratory of Hypoxia Translational Medicine, Xuanwu Hospital, Capital Medical University, Beijing 100053, China. ${ }^{9}$ National Engineering Laboratory of Internet Medical Diagnosis and Treatment TechnologyXuanwu Hospital, Capital Medical University, Beijing 100053, China.

Received: 5 March 2021 Accepted: 17 May 2021

Published online: 24 May 2021

\section{References}

1. Liu G, Bao X, Jiang Y, Liao M, Jiang Q, Feng R, Zhang L, Ma G, Chen Z, Wang $G$, et al. Identifying the association between Alzheimer's disease and Parkinson's disease using genome-wide association studies and protein-protein interaction network. Mol Neurobiol. 2015;52:1629-36.

2. Liu G, Liu Y, Jiang Q, Jiang Y, Feng R, Zhang L, Chen Z, Li K, Liu J. Convergent genetic and expression datasets highlight TREM2 in Parkinson's disease susceptibility. Mol Neurobiol. 2016;53:4931-8.

3. Dias $\mathrm{V}$, Junn E, Mouradian MM. The role of oxidative stress in Parkinson's disease. J Parkinsons Dis. 2013;3:461-91.

4. Heo JH, Hyon L, Lee KM. The possible role of antioxidant vitamin C in Alzheimer's disease treatment and prevention. Am J Alzheimers Dis Other Demen. 2013;28:120-5.

5. Ide K, Yamada H, Umegaki K, Mizuno K, Kawakami N, Hagiwara Y, Matsumoto M, Yoshida H, Kim K, Shiosaki E, et al. Lymphocyte vitamin C levels as potential biomarker for progression of Parkinson's disease. Nutrition. 2015;31:406-8

6. de Rijk MC, Breteler MM, den Breeijen JH, Launer LJ, Grobbee DE, van der Meche FG, Hofman A. Dietary antioxidants and Parkinson disease. The Rotterdam Study. Arch Neurol. 1997;54:762-5.

7. Zhang SM, Hernan MA, Chen H, Spiegelman D, Willett WC, Ascherio A. Intakes of vitamins $E$ and $C$, carotenoids, vitamin supplements, and PD risk. Neurology. 2002;59:1161-9.

8. Miyake Y, Fukushima W, Tanaka K, Sasaki S, Kiyohara C, Tsuboi Y, Yamada T, Oeda T, Miki T, Kawamura N, et al. Dietary intake of antioxidant vitamins and risk of Parkinson's disease: a case-control study in Japan. Eur J Neurol. 2011;18:106-13.

9. Hughes KC, Gao X, Kim IY, Rimm EB, Wang M, Weisskopf MG, Schwarzschild MA, Ascherio A. Intake of antioxidant vitamins and risk of Parkinson's disease. Mov Disord. 2016;31:1909-14.

10. Yang F, Wolk A, Hakansson N, Pedersen NL, Wirdefeldt K. Dietary antioxidants and risk of Parkinson's disease in two population-based cohorts. Mov Disord. 2017:32:1631-6.

11. Hantikainen E, Trolle Lagerros Y, Ye W, Serafini M, Adami HO, Bellocco R, Bonn S. Dietary antioxidants and the risk of Parkinson disease: the Swedish National March Cohort. Neurology. 2021;96:e895-903.

12. Liu G, Zhao Y, Jin S, Hu Y, Wang T, Tian R, Han Z, Xu D, Jiang Q. Circulating vitamin E levels and Alzheimer's disease: a Mendelian randomization study. Neurobiol Aging. 2018;72:189e181-9.

13. Zheng JS, Luan J, Sofianopoulou E, Imamura F, Stewart ID, Day FR, Pietzner M, Wheeler E, Lotta LA, Gundersen TE, et al. Plasma vitamin C and type 2 diabetes: genome-wide association study and Mendelian randomization analysis in European populations. Diabetes Care. 2021:44:98-106.

14. Nalls MA, Blauwendraat C, Vallerga CL, Heilbron K, Bandres-Ciga S, Chang D, Tan M, Kia DA, Noyce AJ, Xue A, et al. Identification of novel risk loci, causal insights, and heritable risk for Parkinson's disease: a meta-analysis of genome-wide association studies. Lancet Neurol. 2019;18:1091-102.

15. Blauwendraat $C$, Heilbron $K$, Vallerga CL, Bandres-Ciga S, von Coelln $R$, Pihlstrom L, Simon-Sanchez J, Schulte C, Sharma M, Krohn L, et al. Parkinson's disease age at onset genome-wide association study: Defining heritability, genetic loci, and alpha-synuclein mechanisms. Mov Disord. 2019:34:866-75. 
16. He Y, Zhang H, Wang T, Han Z, Ni QB, Wang K, Wang L, Zhang Y, Hu Y, Jin S, et al. Impact of serum calcium levels on Alzheimer's disease: a Mendelian randomization study. J Alzheimers Dis. 2020;76:713-24.

17. Zhang H, Wang T, Han Z, Liu G. Mendelian randomization study to evaluate the effects of interleukin- 6 signaling on four neurodegenerative diseases. Neurol Sci. 2020;41:2875-82.

18. Wang L, Qiao Y, Zhang H, Zhang Y, Hua J, Jin S, Liu G. Circulating vitamin D levels and Alzheimer's disease: a Mendelian randomization study in the IGAP and UK Biobank. J Alzheimers Dis. 2020;73:609-18.

19. Shah S, Henry A, Roselli C, Lin H, Sveinbjornsson G, Fatemifar G, Hedman AK, Wilk JB, Morley MP, Chaffin MD, et al. Genome-wide association and Mendelian randomisation analysis provide insights into the pathogenesis of heart failure. Nat Commun. 2020;11:163.

20. Thom CS, Ding Z, Levin MG, Damrauer SM, Lee KM, Lynch J, Chang KM, Tsao PS, Cho K, Wilson PWF, et al. Genetic determinants of increased body mass index mediate the effect of smoking on increased risk for type 2 diabetes but not coronary artery disease. Hum Mol Genet. 2020;29:3327-37.

21. Leong A, Cole JB, Brenner LN, Meigs JB, Florez JC, Mercader JM. Cardiometabolic risk factors for COVID-19 susceptibility and severity: a Mendelian randomization analysis. PLoS Med. 2021;18:e1003553.

22. Zheng J, Baird D, Borges MC, Bowden J, Hemani G, Haycock P, Evans DM, Smith GD. Recent developments in Mendelian randomization studies. Curr Epidemiol Rep. 2017:4:330-45.

23. Simon-Sanchez J, van Hilten JJ, van de Warrenburg B, Post B, Berendse HW, Arepalli S, Hernandez DG, de Bie RM, Velseboer D, Scheffer $\mathrm{H}$, et al. Genome-wide association study confirms extant PD risk loci among the Dutch. Eur J Hum Genet. 2011;19:655-61.

24. Simon-Sanchez J, Schulte C, Bras JM, Sharma M, Gibbs JR, Berg D, PaisanRuiz C, Lichtner P, Scholz SW, Hernandez DG, et al. Genome-wide association study reveals genetic risk underlying Parkinson's disease. Nat Genet. 2009;41:1308-12.

25. Nalls MA, Bras J, Hernandez DG, Keller MF, Majounie E, Renton AE, Saad M, Jansen I, Guerreiro R, Lubbe S, et al. NeuroX, a fast and efficient genotyping platform for investigation of neurodegenerative diseases. Neurobiol Aging. 2015;36(1605):e1607-12.

26. Bandres-Ciga S, Ahmed S, Sabir MS, Blauwendraat C, Adarmes-Gomez AD, Bernal-Bernal I, Bonilla-Toribio M, Buiza-Rueda D, Carrillo F, CarrionClaro $M$, et al. The genetic architecture of parkinson disease in Spain: characterizing population-specific risk, differential haplotype structures, and providing etiologic insight. Mov Disord. 2019;34:1851-63.

27. Spencer CC, Plagnol V, Strange A, Gardner M, Paisan-Ruiz C, Band G, Barker RA, Bellenguez C, Bhatia K, Blackburn H, et al. Dissection of the genetics of Parkinson's disease identifies an additional association $5^{\prime}$ of SNCA and multiple associated haplotypes at 17q21. Hum Mol Genet. 2011:20:345-53.

28. Verbanck M, Chen CY, Neale B, Do R. Detection of widespread horizontal pleiotropy in causal relationships inferred from Mendelian randomization between complex traits and diseases. Nat Genet. 2018;50:693-8.

29. Greco MF, Minelli C, Sheehan NA, Thompson JR. Detecting pleiotropy in Mendelian randomisation studies with summary data and a continuous outcome. Stat Med. 2015:34:2926-40.

30. Liu G, Zhang S, Cai Z, Ma G, Zhang L, Jiang Y, Feng R, Liao M, Chen Z, Zhao B, Li K. PICALM Gene rs3851179 polymorphism contributes to Alzheimer's disease in an Asian Population. Neuromolecular Med. 2013;15:384-8.

31. Burgess S, Thompson SG. Interpreting findings from Mendelian randomization using the MR-Egger method. Eur J Epidemiol. 2017;32:377-89.

32. Shim H, Chasman DI, Smith JD, Mora S, Ridker PM, Nickerson DA, Krauss RM, Stephens M. A multivariate genome-wide association analysis of 10 LDL subfractions, and their response to statin treatment, in 1868 Caucasians. PLoS ONE. 2015;10:e0120758.

33. Brion MJ, Shakhbazov K, Visscher PM. Calculating statistical power in Mendelian randomization studies. Int J Epidemiol. 2013:42:1497-501.

34. King D, Playfer JR, Roberts NB. Concentrations of vitamins A, C and E in elderly patients with Parkinson's disease. Postgrad Med J. 1992;68:634-7.

35. Fernandez-Calle P, Jimenez-Jimenez FJ, Molina JA, Cabrera-Valdivia F, Vazquez A, Garcia Urra D, Bermejo F, Cruz Matallana M, Codoceo R. Serum levels of ascorbic acid (vitamin C) in patients with Parkinson's disease. J Neurol Sci. 1993;118:25-8.

36. Naidu KA. Vitamin C in human health and disease is still a mystery? An overview. Nutr J. 2003;2:7

37. Podmore ID, Griffiths HR, Herbert KE, Mistry N, Mistry P, Lunec J. Vitamin C exhibits pro-oxidant properties. Nature. 1998;392:559.

38. Kazmierczak-Baranska J, Boguszewska K, Adamus-Grabicka A, Karwowski BT. Two faces of Vitamin C-antioxidative and pro-oxidative agent. Nutrients. 2020;12.

39. Michels AJ, Hagen TM, Frei B. Human genetic variation influences vitamin C homeostasis by altering vitamin C transport and antioxidant enzyme function. Annu Rev Nutr. 2013:33:45-70.

\section{Publisher's Note}

Springer Nature remains neutral with regard to jurisdictional claims in published maps and institutional affiliations.
Ready to submit your research? Choose BMC and benefit from:

- fast, convenient online submission

- thorough peer review by experienced researchers in your field

- rapid publication on acceptance

- support for research data, including large and complex data types

- gold Open Access which fosters wider collaboration and increased citations

- maximum visibility for your research: over $100 \mathrm{M}$ website views per year

At BMC, research is always in progress.

Learn more biomedcentral.com/submissions 